\title{
Energia necessária para a ocorrência de fratura em modelos sintéticos de fêmur proximal após retirada de material de síntese: Um estudo biomecânico com parafuso canulado, parafuso dinâmico do quadril e haste femoral proximal*
}

\section{Energy Required for Fracture in Synthetic Proximal Femoral Models after Synthesis Material Removal: A Biomechanical Study Using Cannulated Screws, Dynamic Hip Screws, and Proximal Femoral Nails}

Anderson Freitas ${ }^{10}$ Landwehrle de Lucena da Silva ${ }^{2}$ Renilton Rodrigues Costa ${ }^{2}(0)$

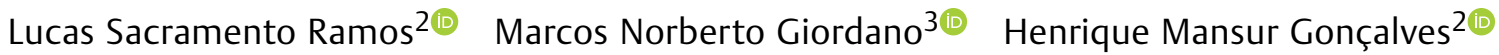

1 Instituto de Pesquisa e Ensino do Hospital Ortopédico e Medicina Especializada (IPE-HOME-DF), Brasília, DF, Brasil

${ }^{2}$ Hospital Regional do Gama, Brasília, DF, Brasil

${ }^{3}$ Hospital de Aeronáutica do Galeão (HFAG), Rio de Janeiro, RJ, Brasil Rev Bras Ortop 2021;56(2):251-255.
Endereço para correspondência Landwehrle de Lucena da Silva, MD, Hospital Regional do Gama, Área Especial n 01, St. Central, Gama, Brasília, DF, 72405-901, Brasil (e-mail: landlucena@gmail.com).
Resumo

Palavras-chave

- quadril

- fraturas do quadril

- fixação de fratura

- remoção de dispositivo
Objetivo Identificar a energia necessária para ocorrência de fratura do fêmur proximal em osso sintético após retirada de três modelos de implantes: parafusos canulados, parafuso dinâmico do quadril (dynamic hip screw-DHS) e haste femoral proximal (proximal femoral nail-PFN).

Métodos Foram utilizados 25 modelos de ossos sintéticos da extremidade proximal do fêmur: 10 unidades de grupo controle (GC), 5 unidades após colocação e retirada de 3 parafusos canulados colocados em configuração de triângulo invertido (GPC), 5 unidades após colocação e retirada do parafuso de compressão dinâmico (GDHS), e 5 unidades após colocação e retirada da haste de fêmur proximal (GPFN). Uma análise biomecânica foi realizada em todas as amostras simulando uma queda sobre o grande
${ }^{*}$ Estudo Desenvolvido Pelo Serviço de Ortopedia e traumatologia do Hospital Regional do Gama, Brasília-DF e pelo Instituto de Pesquisa e Ensino do Hospital Ortopédico e Medicina especializada (IPE-HOME), Brasília, DF, Brasil. recebido

17 de Fevereiro de 2020

aceito

16 de Setembro de 2020
DOI https://doi.org/

10.1055/s-0040-1721832. ISSN 0102-3616. (c) 2021. Sociedade Brasileira de Ortopedia e Traumatologia. All rights reserved.

This is an open access article published by Thieme under the terms of the Creative Commons Attribution-NonDerivative-NonCommercial-License, permitting copying and reproduction so long as the original work is given appropriate credit. Contents may not be used for commercial purposes, or adapted, remixed, transformed or built upon. (https://creativecommons.org/ licenses/by-nc-nd/4.0/)

Thieme Revinter Publicações Ltda., Rua do Matoso 170, Rio de Janeiro, RJ, CEP 20270-135, Brazil 


\begin{abstract}
Keywords

- hip

- hip fractures

- fracture fixation

- device removal

Objective The present study aims to identify the energy required for synthetic proximal femoral fracture after removal of three implant types: cannulated screws (CSs), dynamic hip screws (DHSs) and proximal femoral nail (PFNs).

Methods Twenty-five synthetic proximal femur bones were used: 10 were kept intact as the control group (CG), 5 were submitted to the placement and removal of three cannulated screws in an inverted triangle configuration (CSG), 5 were submitted to the placement and removal of a dynamic compression screw (DHSG), and 5 were submitted to the placement and removal of a proximal femur nail (PFNG). All samples were biomechanically analyzed simulating a fall on the greater trochanter using a servo-hydraulic machine to determine the energy (in Joules [J]) required for fracture. Results All samples presented basicervical fractures. The energy required for fracture was $7.1 \mathrm{~J}, 6.6 \mathrm{~J}, 6 \mathrm{~J}$ and 6.7 J for the CG, CSG, DHSG, and PFNG, respectively. There was no statistically significant difference (considering a $95 \%$ confidence interval) in energy among the study groups $(p=0.34)$.

Conclusion There was no statistically significant difference in the energy required to cause a synthetic proximal femoral fracture after removing all three implant types and simulating a fall over the greater trochanter.
\end{abstract}

trocânter utilizando uma máquina servo-hidráulica com o objetivo de verificar a energia (em Joules [J]) necessária até a ocorrência de fratura nos diferentes grupos.

Resultados Todos os grupos apresentaram fratura basocervical. Os grupos GC, GPC, GDHS e GPFN apresentaram, respectivamente, valores de 7.1], 6.6], 6] e 6.7] de energia até ocorrência da fratura. Não houve diferença estatisticamente significativa (intervalo de confiança de $95 \%)$ na energia entre os grupos de estudo $(p=0,34)$.

Conclusão Não houve diferença estatisticamente significativa nos valores de energia necessária para ocorrência de fratura da extremidade proximal do fêmur após a retirada de três tipos de implantes utilizando modelos sintéticos simulando queda sobre o grande trocânter.

\section{Introdução}

A expectativa de vida populacional tem aumentado em todo mundo, sobretudo pela melhora dos determinantes sociais de saúde. Consequentemente, o número de idosos tem aumentado, proporcionalmente, a taxa de doenças crônicas não transmissíveis. Dentre elas, a osteoporose se destaca por ter se tornado um problema de saúde pública de ocorrência global. Acomete majoritariamente a população idosa, em especial os pacientes do sexo feminino após a menopausa. É caracterizada pela redução da densidade mineral óssea e, consequentemente, redução da resistência mecânica do osso. Tem um impacto socioeconômico importante devido à alta incidência de fraturas do fêmur proximal resultantes de quedas e traumas de baixa energia., ${ }^{1,2}$

A abordagem dessas fraturas consiste em fornecer ao paciente condições de retomada das atividades habituais o mais precocemente possível. Por isso, na maioria das vezes realiza-se o tratamento cirúrgico com colocação de implantes, como as hastes femorais (PFNs-proximal femoral nails), parafusos canulados, placa tubo deslizante (DHS-dynamic hip screw), ou ainda por meio de substituição articular (artroplastia). ${ }^{3}$

Algumas complicações associadas ao tratamento cirúrgico das fraturas da extremidade proximal do fêmur podem levar à necessidade de retirada do implante. As indicações para retirada do material de síntese são principalmente por dor persistente no quadril, glúteo ou coxa, ou ainda devido à falha ou infecção do implante. ${ }^{4-6} \mathrm{~A}$ remoção de implantes pode predispor fraturas do colo femoral ou da região intertrocantérica, principalmente em pacientes idosos com baixa qualidade óssea. $^{7}$

Em função das várias dimensões e diferentes posicionamentos dos implantes utilizados nas fraturas do fêmur proximal, existe a necessidade de entender quais as implicações biomecânicas resultantes de sua retirada, e, desta maneira, conscientizar o cirurgião sobre a segurança e as consequências da realização desse procedimento. ${ }^{8,9}$

Para isto, este estudo tem como objetivo identificar a energia necessária (em Joules [J]) para a ocorrência de fratura do fêmur proximal em osso sintético após a remoção de três modelos de implantes: parafusos canulados, parafuso dinâmico do quadril (DHS) e haste proximal do fêmur (PFN).

\section{Materiais e Métodos}

Foram utilizados 25 fêmures sintéticos (modelo c1010 da fabricante Nacional Ossos, Jaú-SP, Brasil) compostos por osso 


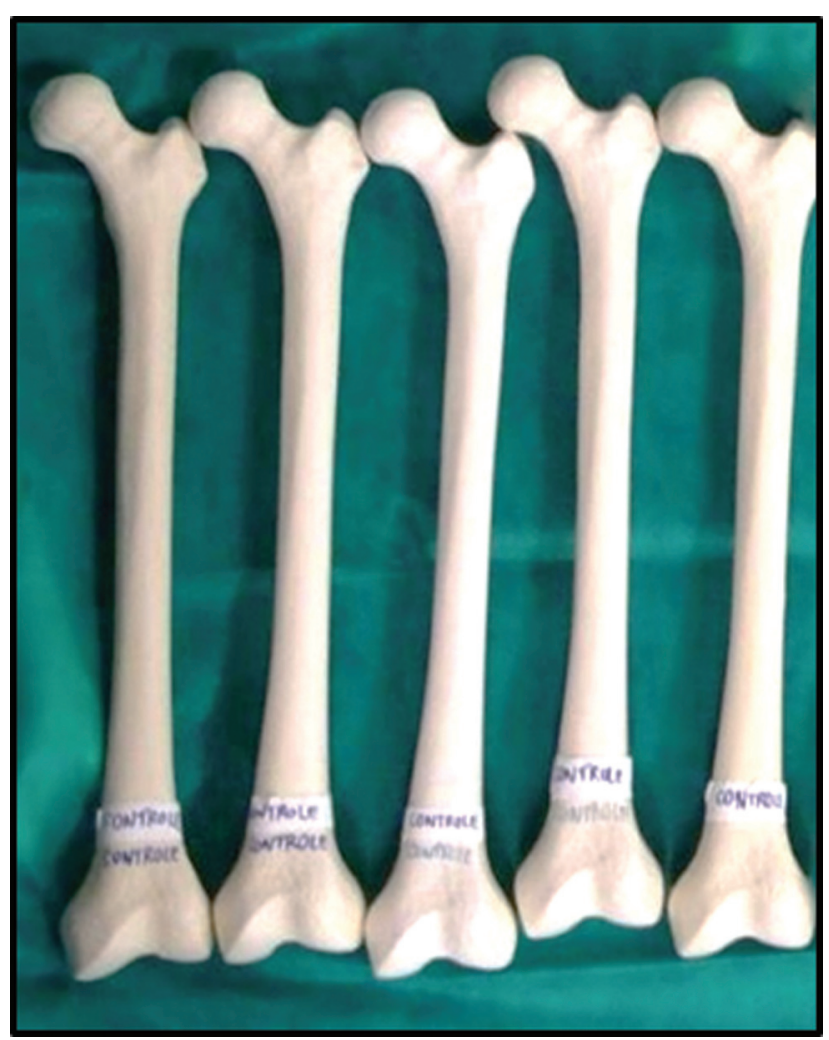

Fig. 1 Amostra de modelos do grupo controle (GC).

cortical e esponjoso, com 10 pfc (pounds per cubic foot) e canal medular de $12 \mathrm{~mm}$. Em seguida, estes fêmures foram divididos em quatro grupos: grupo controle (GC), grupo parafuso canulado (GPC), grupo dynamic hip screw (GDHS) e grupo proximal femoral nail (GPFN).

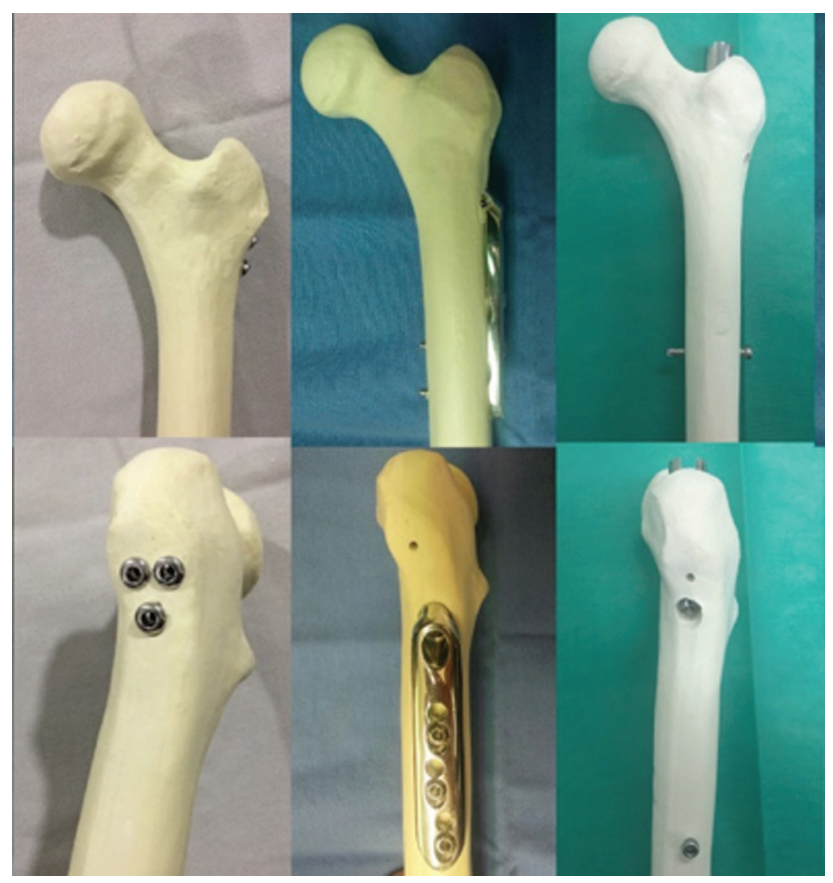

Fig. 2 Amostra de modelos dos grupos GPC, GDHS, e GPFN após colocação do implante.

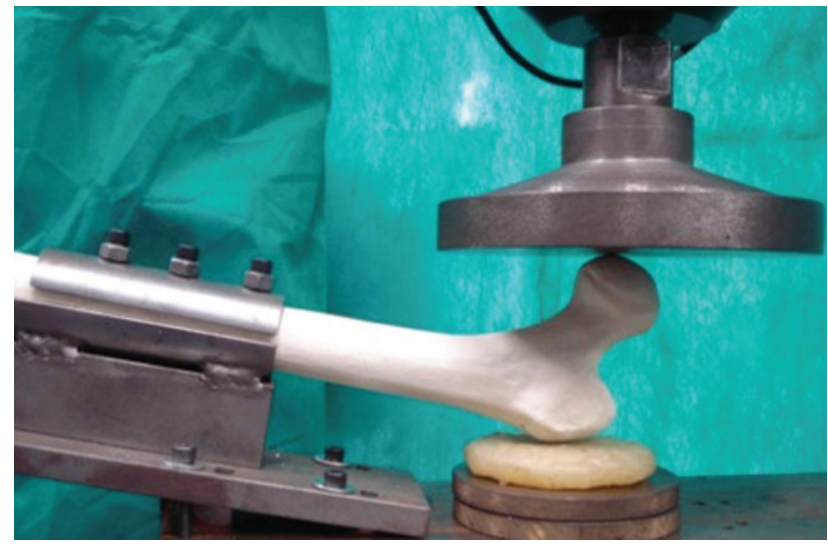

Fig. 3 Modelo experimental na plataforma de ensaio biomecânico.

O GC foi formado por 10 fêmures intactos (-Figura 1). No grupo GPC, 5 fêmures sintéticos intactos foram submetidos à colocação de três parafusos canulados de $7,5 \mathrm{~mm}$ configurados em triângulo invertido. Os grupos GDHS e GPFN foram formados cada um por 5 fêmures sintéticos e fixados com seus implantes conforme descrito pela técnica AO (Arbeitsgemeinschaft für Osteosynthesefragen), conforme a - Figura 2. O diâmetro dos parafusos deslizantes era de $12 \mathrm{~mm}$ no grupo GDHS e $10,5 \mathrm{~mm}$ no grupo GPFN. Por fim, retirou-se todos os implantes e os ossos foram encaminhados para o laboratório de análise biomecânica.

Os ensaios foram feitos de forma estática em flexão utilizando a máquina servo-hidráulica do modelo MTS 810-FlexTest 40 (MTS Sistemas do Brasil Ltda., São Paulo, $\mathrm{SP}$, Brasil) com capacidade de 100 kilonewtons ( $\mathrm{kN})$. $\mathrm{O}$ fêmur foi fixado ao dispositivo de ensaio deixando $150 \mathrm{~mm}$ do seu comprimento fora do dispositivo em direção ao pistão hidráulico, posicionado na base da máquina de ensaio com inclinação de $10^{\circ} \mathrm{com}$ a horizontal e $15^{\circ}$ de rotação interna aferidos com goniômetro digital. 0 trocânter maior mantevese apoiado em um disco de silicone de $8 \times 2 \mathrm{~cm}$ de diâmetro (-Figura 3). Foi aplicada uma pré-carga de $40 \mathrm{~N}$ (newtons) a uma velocidade de $2 \mathrm{~mm} / \mathrm{s}$ e em seguida aplicada uma carga

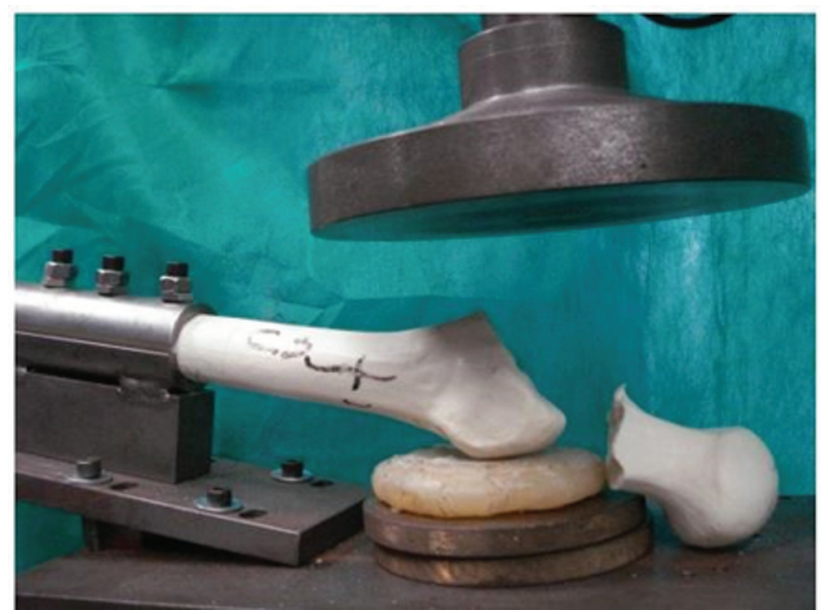

Fig. 4 Modelo experimental após ocorrência de fratura. 
Tabela 1 Parâmetros de energia (J) necessária até ocorrência de fratura nos modelos experimentais

\begin{tabular}{|l|l|l|l|l|l|l|}
\hline Variável & $\mathbf{n}$ & média & IC 95\% para média & mínima & máxima & Valor de $\boldsymbol{p}^{*}$ \\
\hline Energia (J) & & & & & & \\
\hline GC & 10 & 7,1 & $5.5-8,6$ & 4,4 & 10,4 & \\
\hline GPC & 5 & 6,6 & $4.3-8,9$ & 4 & 10 & \\
\hline GDHS & 5 & 6 & $4.9-7,1$ & 4 & 7 & \\
\hline GPFN & 5 & 6,7 & $6.1-7,3$ & 6,2 & 7,9 & 0,78 \\
\hline
\end{tabular}

Abreviaturas: GC, grupo controle; GDHS, grupo dynamic hip screw (parafuso dinâmico do quadril); GPC, grupo parafuso canulado; GPFN, grupo proximal femoral nail (haste femoral proximal); IC, intervalo de confiança; J, Joules.

${ }^{*}$ ANOVA one-way: análise de variância simples.

na cabeça do fêmur até ocasionar fratura (-Figura 4), obtendo-se os valores de energia em J.

Os resultados foram obtidos por meio de uma análise inferencial utilizando os valores dos parâmetros selecionados, compostos pela análise de variância simples (ANOVA one-way), com o objetivo de verificar a existência de diferença significativa entre os grupos GC, GPC, GDHS e GPFN. O nível de significância foi de $5 \%$. A análise estatística foi realizada pelo software IBM Statistical Package for the Social Sciences (SPSS) Statistics, versão 20.0 (IBM SPSS Statistics, Armonk, NY, EUA).

\section{Resultados}

Todos os grupos apresentaram fratura do fêmur proximal do tipo basocervical.

Os grupos apresentaram valores de energia média para a fratura em J, respectivamente: GC 7.1J, GPC 6.6J, GDHS 6J e GPF 6.7J conforme - Tabela $\mathbf{1}$.

Observou-se, segundo a ANOVA one-way, que não existe diferença significativa, na energia exigida até fratura $(p=0,78)$ entre os grupos de estudo.

\section{Discussão}

A retirada de implantes do fêmur proximal pode gerar alterações biomecânicas locais. O DHS, por exemplo, pode gerar defeitos ósseos na área subtrocantérica em função de seu posicionamento; enquanto a retirada do PFN gera um defeito ósseo maior na área do grande trocânter. Por isso, antes da retirada de implantes do fêmur proximal, o cirurgião deve considerar as alterações biomecânicas e as possíveis complicações resultantes. ${ }^{7-9}$

Neste estudo, a escolha de ossos sintéticos teve como objetivo a uniformização das propriedades biomecânicas entre as amostras e minimizar as diferenças inerentes aos ossos humanos (densidade óssea, comprimento, composição bioquímica, idade, diâmetro). ${ }^{10} \mathrm{O}$ mecanismo de fratura simulado, a queda sobre o grande trocânter, é aceito como o de maior ocorrência neste tipo de fratura, principalmente na população idosa. ${ }^{11}$

Todas as fraturas encontradas em nosso estudo foram do tipo basocervical. A literatura sugere que após a remoção do implante, a falha óssea agravada pela baixa densidade óssea em pacientes idosos pode contribuir para o enfraquecimento da região do colo femoral, tornando-o mais susceptível a concentração de estresse e a ocorrência de fratura nesta região. ${ }^{12-14}$ Outros estudos têm sugerido que as dores relatadas pelo paciente mesmo após a consolidação da fratura e previamente a retirada do implante, podem ter sido interpretadas incorretamente e que na realidade seriam sinais clínicos de fratura por estresse na região do colo femoral, o que contribuiria para ocorrência de fratura após retirada do implante. $^{15}$

Os resultados ainda permitiram observar um comportamento regular de tendência de redução dos valores da energia máxima nos GPC, GDHS, GPFN quando comparados ao grupo controle (GC), ainda que não houvesse diferença significativa do ponto de vista estatístico. Yang e Cols, através de um estudo biomecânico similar utilizando 15 fêmures cadavéricos, também não conseguiram demonstrar diferença significativa nos valores de energia máxima para ocorrência de fratura no fêmur proximal após retirada de implantes do tipo PFN e DHS.,

Outros estudos testaram o uso do reforço femoral com cimento ósseo como técnica de proteção à fratura do fêmur proximal em ossos osteoporóticos após retirada de material de síntese. Num desses estudos, utilizando fêmures sintéticos divididos em 2 grupos, um com e outro sem reforço de cimento ósseo após retirada do implante (DHS), realizaram testes biomecânicos com intuito de testar parâmetros de energia máxima até ocorrência de fratura. De maneira curiosa, não foi encontrado diferença estatística nos valores de energia máxima até a fratura, o que sugere que o efeito da cimentação após retirada do implante não traria benefício. ${ }^{8,16}$

No que se refere às limitações do estudo, (1) percebemos que a carga exercida nos modelos foi essencialmente uma força de compressão lateral pura, embora outras variáveis, como a força rotacional e axial, possam atuar sobre o fêmur in vivo. (2) Outra limitação importante foi a utilização do modelo ósseo sintético. Sabemos que ele não reproduz a verdadeira biomecânica do osso humano, principalmente na população idosa, que tem baixa densidade mineral óssea e que mais sofre fratura do fêmur proximal. Além disto, as alterações morfológicas próprias da cicatrização da fratura, como a formação de calo, o remodelamento e a má união não foram avaliadas. A raça, idade, doenças metabólicas e hábitos de vida são outras variáveis não avaliadas nos modelos sintéticos utilizados. Não obstante, a própria agressão iatrogênica necessária para remoção do material também não 
pode ser avaliada. (3) Por fim, a amostra restrita a 25 ossos mostrou-se limitada devido ao alto custo dos modelos.

\section{Conclusão}

Entre os modelos ósseos avaliados, não encontramos diferenças significativas nos valores de energia para ocorrência de fratura quando comparados ao GC. Há necessidade de mais estudos que corroborem os resultados, preferencialmente com modelos ósseos que se assemelhem biomecanicamente aos da população com maior incidência de fratura do fêmur proximal.

Conflito de Interesses

Os autores não têm conflito de interesses a declarar.

\section{Referências}

1 Cosman F, de Beur SJ, LeBoff MS, et al. National Osteoporosis Foundation. Clinician's Guide to Prevention and Treatment of Osteoporosis. Osteoporos Int 2014;25(10):2359-2381

2 Burge R, Dawson-Hughes B, Solomon DH, Wong JB, King A, Tosteson A. Incidence and economic burden of osteoporosisrelated fractures in the United States, 2005-2025. J Bone Miner Res 2007;22(03):465-475

3 Rozell JC, Hasenauer M, Donegan DJ, Neuman M. Recent advances in the treatment of hip fractures in the elderly. F1000 Res 2016;5: F1000

4 Holley K, Zelken J, Padgett D, Chimento G, Yun A, Buly R. Periprosthetic fractures of the femur after hip arthroplasty: an analysis of 99 patients. HSS J 2007;3(02):190-197

5 Onche II, Osagie OE, INuhu S. Removal of orthopaedic implants: indications, outcome and economic implications. J West Afr Coll Surg 2011;1(01):101-112
6 Hernandez AJ, Fávaro E, Laraya MH, Zumiotti AV. Fratura espontânea do colo do fêmur após retirada de PFN. Acta Ortop Bras 2009;17(03):187-189

7 Kovar FM, Strasser E, Jaindl M, Endler G, Oberleitner G. Complications following implant removal in patients with proximal femur fractures - an observational study over 16 years. Orthop Traumatol Surg Res 2015;101(07):785-789

8 Eberle S, Wutte C, Bauer C, von Oldenburg G, Augat P. Should extramedullary fixations for hip fractures be removed after bone union? Clin Biomech (Bristol, Avon) 2011;26(04):410-414

9 Yang JH, Jung TG, Honnurappa AR, et al. The Analysis of Biomechanical Properties of Proximal Femur after Implant Removal. Appl Bionics Biomech 2016;2016:4987831

10 Cristofolini L, Viceconti M, Cappello A, Toni A. Mechanical validation of whole bone composite femur models. J Biomech 1996; 29(04):525-535

11 Parkkari J, Kannus P, Palvanen M, et al. Majority of hip fractures occur as a result of a fall and impact on the greater trochanter of the femur: a prospective controlled hip fracture study with 206 consecutive patients. Calcif Tissue Int 1999;65(03):183-187

12 Taylor PR, Hepple S, Stanley D. Combination subcapital and intertrochanteric fractures of the femoral neck. Injury 1996;27 (01):68-71

13 Rosson J, Murphy W, Tonge C, Shearer J. Healing of residual screw holes after plate removal. Injury 1991;22(05):383-384

14 Buciuto R, Hammer R, Herder A. Spontaneous subcapital femoral neck fracture after healed trochanteric fracture. Clin Orthop Relat Res 1997;(342):156-163

15 Paiva LM, Macedo Neto SL, Souto DRM, Ferreira GNB, Costa HID, Freitas A. Static bending test after proximal femoral nail (PFN) removal - in vitro analysis. Rev Bras Ortop 2017;52(Suppl 1): 52-56

16 Freitas A, Breta JB, Júnior J, et al. Biomechanical Test Following Removal of a Dynamic Hip Screw: In Vitro Analysis. Cureus 2018; 10(12):e3680 